\author{
Corpus \\ CORPUS \\ $22 \mid 2021$ \\ Du recueil à l'outillage des corpus oraux : comment \\ accéder à la variation?
}

\title{
Corpus ESLO-Enfants : de sa création aux premiers résultats
}

Jennifer Ganaye

\section{(2) OpenEdition \\ Journals}

Édition électronique

URL : https://journals.openedition.org/corpus/6161

DOI : $10.4000 /$ corpus.6161

ISSN : 1765-3126

Éditeur

Bases; corpus et langage - UMR 6039

Référence électronique

Jennifer Ganaye, "Corpus ESLO-Enfants : de sa création aux premiers résultats », Corpus [En ligne],

22 | 2021, mis en ligne le 15 février 2021, consulté le 05 mars 2022. URL : http://

journals.openedition.org/corpus/6161 ; DOI : https://doi.org/10.4000/corpus.6161

Ce document a été généré automatiquement le 5 mars 2022

(c) Tous droits réservés 


\title{
Corpus ESLO-Enfants : de sa création aux premiers résultats
}

\author{
Jennifer Ganaye
}

\section{Origines du corpus ESLO-Enfants}

1 L'initiative de mettre au point un corpus intégrant dans un même projet la parole des enfants, la parole de leur entourage et la prise en compte de l'environnement dans lequel les échanges ont lieu trouve son origine dans notre volonté d'élargir le périmètre actuel des études de corpus enfantins en français, mais aussi d'étudier, l'impact de l'input sur l'output en fonction de différents environnements langagiers.

2 Les nombreux travaux menés dans le cadre des Théories Basées sur l'Usage (UBT) mettent en évidence l'importance de l'environnement (ou input) des enfants pour l'apprentissage de leur(s) langue(s) (Tomasello, 2003). Cet input constituant d'une certaine façon la matière première (Bybee \& Hopper, 2001) à partir de laquelle l'enfant va pouvoir construire la grammaire de sa langue, développer son lexique. Dès le plus jeune âge, l'enfant reçoit une grande quantité de paroles de la part de son entourage qui l'aidera à développer ses compétences lexicales, dont une partie provient des scènes d'attention conjointe (Goldwin-Meadow, 2010).

Pour l'heure, les corpus enfantins en français (citons par exemple : CoLaJE, ALIPE, TCOF et Corpus de Lyon) ne peuvent rendre compte de cela que de façon parcellaire. Nous constatons que les enfants participants à ces différents projets sont souvent issus de milieux favorisés, voire très favorisés. Cela est souvent dû à la facilité de trouver des familles ayant un niveau socio-économique plus élevé dans l'entourage du chercheur. Mais qu'en serait-il avec des enfants évoluant dans un milieu moins favorisé ? C'est en cela que le module ESLO-enfants se veut novateur et original puisque nous souhaitons élargir le cercle des enfants enregistrés en veillant à intégrer, dans notre échantillon, des enfants issus de milieux sociaux variés à l'image de ce que peut être la population d'une ville. Constituer un tel panel permettra d'accéder à des interactions familiales 
pour lesquelles nous n'avons pour l'heure que très peu de données et peut-être de questionner l'influence des milieux socio-économiques sur l'usage du langage.

En effet, s'inscrire dans une approche où l'input est au centre de la construction du langage a des conséquences théoriques et méthodologiques fortes. Dans ce cadre, nous empruntons certains principes de la linguistique variationniste (Labov, 1976) qui sont en totale cohérence avec ceux des UBT (Kemmer \& Barlow, 2000).

Il faut aussi prendre en compte que notre corpus fait partie d'un projet de laboratoire plus large de recueil, documentation et mise à disposition d'un grand corpus de français parlé (Enquêtes SocioLinguistiques à Orléans : ESLO) collecté à deux périodes : dans les années 1970 et dans les années 2010. Dès la première phase de collecte, les chercheurs avaient pour objectif de créer un corpus de type sociolinguistique :

Selon nous une recherche sociolinguistique impliquait une étude de la langue dans sa diversité plutôt que comme un tout homogène et figé. En effet, même si on étudie un état de langue à un moment précis de l'histoire, il n'empêche qu'il offre une variété à plusieurs niveaux: différences entre les générations; différences dialectales entre communautés; différences entre les milieux sociaux; différences liées aux conditions de production du discours. (Blanc \& Biggs, 1971 : 16)

Par la suite, l'approche variationniste a orienté le travail des chercheurs, en mettant notamment en avant la prise en compte de l'hétérogénéité des pratiques langagières, et en cherchant à rendre compte de la structure des variations (Abouda \& Baude, 2005). L'importance accordée aux données et à la possibilité de faire varier les contextes/ situations a grandement guidé notre travail. Nous empruntons à ESLO également sa chaîne de traitement de corpus.

Dans ce corpus variationniste, nous centrerons notre étude sur un phénomène linguistique: l'usage des liaisons nous permettant d'observer ou non l'influence de l'input sur les productions enfantines, point sur lequel nous reviendrons plus en détail par la suite.

\section{Apports du corpus ESLO-Enfants}

8 Les apports du corpus ESLO-Enfants se situent à deux niveaux. Ce module constitue en premier lieu un apport pour ESLO. Nous pouvons en effet constater qu'aujourd'hui, dans ESLO, il n'y a pas de données contenant des paroles d'enfants en interaction. Précisons toutefois l'existence de deux modules qui intègrent des enfants :

9 - le module "Ecole», où il est possible d'entendre des enfants, mais ces enregistrements ne sont pas focalisés sur la parole enfantine, mais plutôt sur l'ensemble de la vie d'une école.

10 - le module "Livres pour enfants » en cours de traitement, dans lequel l'objectif était d'enregistrer des moments de lecture faits à des enfants, les lecteurs pouvaient être les parents ou bien des personnes de l'entourage proche des enfants. Dans ce dernier, les enregistrements sont focalisés sur les paroles de l'adulte et non celles des enfants.

11 Notre corpus élargit le périmètre actuel d'ESLO avec de nouveaux locuteurs d'Orléans qui représentent une partie non négligeable de la population de la ville.

12 Par ailleurs, nous ajoutons également un nouvel horizon aux corpus enfantins francophones déjà existants, notre corpus comprenant une grande variété de situations d'enregistrement, tout en diversifiant les personnes avec lesquelles l'enfant est amené 
à échanger : par exemple, lorsque l'enfant est amené à passer du temps avec ses grandsparents que ce soit chez eux ou bien chez l'enfant, mais aussi dans sa classe. Nous cherchons donc à obtenir des scènes du quotidien où l'enfant peut jouer avec des amis, sa famille, etc. ou encore lorsqu'il est gardé par une baby-sitter. Nous ne nous restreignons pas aux moments de loisirs, nous captons également des moments simples mais qui font partie d'un quotidien rythmé par des habitudes comportementales et langagières. Ainsi, l'enregistrement de scènes contenant les repas en famille, le bain ou encore le coucher peut être primordial puisque ces dernières contiennent un lexique récurrent où l'enfant pourrait alors acquérir des routines langagières spécifiques. Notre but étant de nous rapprocher au mieux des conversations que pourrait avoir quotidiennement un enfant, pour pouvoir étudier ses différentes sources d'input.

Pour résumer, la création d'un module aussi varié va nous permettre de mieux connaître les influences que les différences socioculturelles peuvent avoir sur l'acquisition de la langue. Nous approfondirons ce point par l'étude du lien input/ output à travers la diffusion de l'usage de la liaison. Notre projet permet donc de compléter le corpus ESLO2 avec des locuteurs qui ne sont que marginalement présents à l'heure actuelle, et dans l'esprit variationniste d'ESLO en veillant à prendre en compte des niveaux de variations différents tout en complétant les corpus enfantins francophones existants.

\section{Objet d'étude : la liaison}

Pour observer la question du lien entre input et output, nous décrirons l'usage des liaisons à la fois dans l'environnement langagier des enfants et dans leurs premières productions. À l'image du travail mené par Liégeois (2014), nous chercherons à rendre compte de l'impact de l'environnement langagier sur les formes produites par les enfants et sur sa propre courbe d'acquisition par rapport au modèle de Chevrot et al. (2009).

\subsection{La liaison : définition}

La liaison est un phénomène oral variable typique du français. Il s'agit de l'oralisation de la consonne finale d'un Mot1 devant un Mot2 à voyelle initiale, consonne muette quand le Mot1 est produit en finale d'énoncé ou devant un mot à initiale consonantique (Soum-Favaro et al. 2014 ; Chevrot et al. 2007) :

$$
\begin{aligned}
& \text { 1. le gros éléphant } \\
& \text { [lagbozelefã } \\
& \text { 2. le gros camion } \\
& \text { [lagbokamjõ] } \\
& \text { 3. il est gros } \\
& \text { [ilegচo] }
\end{aligned}
$$

Dans les cas ci-dessus, nous voyons que le « $\mathrm{s}$ » de «gros » se prononce sous la forme d'une liaison en /z/ uniquement lorsqu'il précède un mot à initiale vocalique (1).

Plusieurs études de corpus avec des méthodes différentes et sur des corpus différents (Corpus d'Orléans: De Jong 1994, ESLO : Dugua \& Baude 2017, PFC: Durand \& Lyche 2008, Coquillon et al. 2010, Durand et al. 2011) décrivent d'ailleurs les variations 
d'usages des liaisons chez l'adulte. Toutes s'accordent sur le fait qu'il existe au moins deux types de liaisons:

18 - Des liaisons systématiques/invariables (classiquement appelées Liaisons obligatoires LO) : les liaisons toujours réalisées quels que soient le locuteur et la situation (De Jong, 1994), exemple :

- après déterminant (des [z] enfants)

- entre pronom personnel et verbe (ils $[z]$ appellent)

- entre verbe et pronom (prends $[z]$ en)

19 - Des liaisons variables ou facultatives (LF) : exemple : « un gros [z] éléphant », qui peut être réalisée ou non. Ce type de liaison est considéré comme une variable sociolinguistique du français dans le sens où, comme le précise Gadet (1989 : 71), elle "permet [à elle seule] de classer socialement un locuteur». L'usage des liaisons variables est conditionnée par des facteurs linguistiques comme les catégories grammaticales des mots, la longueur des mots, la nature de la consonne de liaison, etc. et des facteurs extralinguistiques (ou sociaux) comme le milieu social, les situations de communications (Gadet, 1989). Dans le cas du français, la langue des classes favorisées correspond, dans les esprits, à la norme ou la langue standard (Gadet, 1997), il est donc possible de distinguer des personnes issues de classes différentes par leurs pratiques langagières. Pour les liaisons, par exemple, les classes favorisées ont tendance à plus prononcer les liaisons facultatives (Encrevé, 1988).

\subsection{La liaison : phénomène linguistique percutant}

Pour étudier le lien input/output, s'appuyer sur un objet qui peut être à la fois stable et variable nous fournit un cadre d'analyse riche. Nous pourrons observer par exemple si, ce qui est stable/invariable chez l'adulte, l'est aussi chez l'enfant et à partir de quel âge ; si ce qui est stable est reçu par tous les enfants de la même manière, et ce qui peut différer (la quantité de liaisons stables, la diversité des liaisons stables). Quant aux liaisons variables nous étudierons comment elles varient d'une famille à l'autre, d'un enfant à l'autre et d'une situation à l'autre.

21 Notre approche est fondamentalement basée sur l'usage, sur des données de parole recueillies, et non sur des conceptions normatives ou basées sur des exemples. En étudiant l'usage des liaisons à la fois dans la parole adulte et dans la parole enfantine et en décrivant ce que l'enfant peut récupérer, et cela dans différentes familles ayant un rapport à la langue varié, nous pourrons voir comment la variation se réalise (côté adultes) et se construit (côté enfant) en perception et en production.

Pour convenir à la démarche variationniste recherchée et pour répondre à nos objectifs sur le plan de l'acquisition du langage, nous avons mis en place une procédure de collecte particulière pour accéder au quotidien des enfants de la façon la moins invasive possible, procédure que nous présentons dans la section suivante.

\section{Méthodologie d'enquête}

Rappelons que le corpus ESLO-Enfants s'inscrit dans le plus grand corpus de français parlé en France, le corpus ESLO (http://eslo.huma-num.fr/). Nous avons donc suivi le cadre méthodologique de ce projet tout en l'adaptant à notre nouveau public enfantin. 
Tout d'abord, l'architecture du corpus ESLO (Baude \& Dugua, 2011), à savoir la façon dont les différents modules coexistent et la forme que cette construction donne au corpus, est le reflet $\mathrm{du}$ « parti pris variationniste des auteurs d'ESLO1 et ESLO2 [...] afin de permettre la prise en compte de différents types de variations " (Baude \& Dugua, 2015 :357). Dans cette architecture, le corpus ESLO-Enfants constitue un module particulier puisque nous l'envisageons comme un modèle réduit d'ESLO dans le sens où notre objectif est d'intégrer en son sein la diversité des situations auxquelles un enfant participe au quotidien. Nous cherchons en effet par la constitution de ce corpus à balayer une large palette de situations familières des enfants, sachant toutefois que les contraintes liées à la collecte limiteront les possibilités.

\subsection{Présentation des familles : caractéristiques sociales} trois grands axes: l'axe formation(s)-emploi(s), l'axe temps libre (sorties) et l'axe médias. Ainsi, nous proposons une échelle socio-linguistico-culturo-professionnelle (SLCP), pour classer les différentes familles.

28 À l'aide de ces trois axes, nous avons établi une fiche (voir un extrait ci-dessous) déclinant les principales particularités de chacun des axes. Chaque parent a rempli la fiche informative en précisant des échelles qui varient de 1 à 10. Ainsi, les parents inscrivent eux-mêmes la proportion que chaque axe représente dans leur quotidien. Pour cela, nous nous sommes fondée en partie sur les travaux de Bourdieu (1994) sur la théorie du monde social, et l'avons adapté à nos exigences en termes de public visé et d'informations sur leur input quotidien. Pour chaque axe, nous prenons en compte le temps et la fréquence des activités pratiquées. 


\section{Extrait de la fiche informative}

Culture/Loisirs : A quelle fréquence y allez-vous?

\begin{tabular}{|c|c|c|c|c|c|c|c|c|c|c|}
\hline \multicolumn{11}{|c|}{ Spectacles } \\
\hline 0 & 1 & 2 & 3 & 4 & 5 & 6 & 7 & 8 & 9 & 10 \\
\hline \multicolumn{11}{|c|}{ Cinéma } \\
\hline 0 & 1 & 2 & 3 & 4 & 5 & 6 & 7 & 8 & 9 & 10 \\
\hline \multicolumn{11}{|c|}{ Concert } \\
\hline 0 & 1 & 2 & 3 & 4 & 5 & 6 & 7 & 8 & 9 & 10 \\
\hline \multicolumn{11}{|c|}{ Festivals } \\
\hline 0 & 1 & 2 & 3 & 4 & 5 & 6 & 7 & 8 & 9 & 10 \\
\hline \multicolumn{11}{|c|}{ Théâtre } \\
\hline 0 & 1 & 2 & 3 & 4 & 5 & 6 & 7 & 8 & 9 & 10 \\
\hline \multicolumn{11}{|c|}{ Musées } \\
\hline 0 & 1 & 2 & 3 & 4 & 5 & 6 & 7 & 8 & 9 & 10 \\
\hline \multicolumn{11}{|c|}{ Médiathèque } \\
\hline 0 & 1 & 2 & 3 & 4 & 5 & 6 & 7 & 8 & 9 & 10 \\
\hline
\end{tabular}

Dans l'axe formation(s)-emploi(s), nous faisons apparaitre la/les formation(s) que les parents des familles participantes ont suivies, les éventuelles reprises d'études, le lieu d'étude (pays, région, ville). De la même manière, nous prendrons en compte les emplois qu'ont effectués les parents, la période durant laquelle ils les ont effectués mais aussi, si c'est un travail solitaire ou bien en équipe.

L'axe du temps libre concerne toutes les sorties et activités que les participants peuvent effectuer. Nous les regroupons dans différentes catégories : les sorties culturelles (telles que le théâtre, le cinéma, etc.), les sorties sociales (retrouver des amis pour boire un verre, aller au restaurant, etc.) et des sorties et activités qui comprennent les activités extra-professionnelles (concerne les activités en club mais aussi si les personnes pratiquent des activités à la maison comme par exemple le tricot, les jeux avec leurs enfants, etc.).

Le troisième axe concerne le rapport aux médias. Dès 1950, Queneau faisait remarquer que les médias influencent fortement notre manière de nous exprimer. Ainsi, depuis l'arrivée de la télévision, les individus calquent leur manière de s'exprimer sur celle des présentateurs de télévision car cette forme de langue est jugée plus légitime (Queneau, 1950). Au-delà du petit écran avec le type d'émission que les participants regardent, nous allons nous intéresser aux types de radio, et de lectures, mais aussi la question des usages des vidéos et jeux vidéo, des réseaux sociaux.

Voici une des représentations sous forme de radars obtenues avec le questionnaire que nous venons de décrire. Les exemples exposés ci-dessous concernent les familles des enfants que nous étudierons dans la suite de cet article. 
Figure 1. Représentation des pratiques culturelles des parents de Lucile et Camille

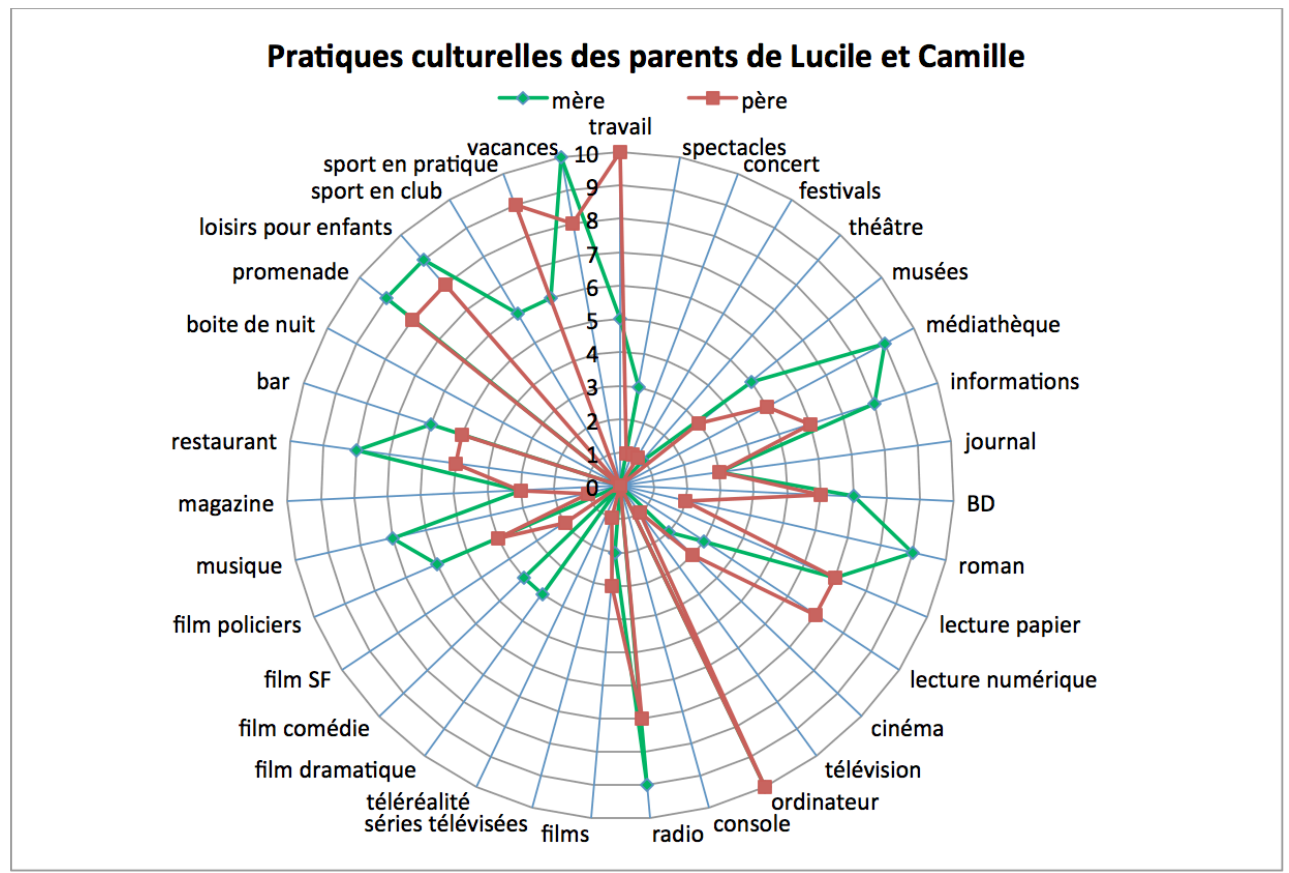

Les tracés révèlent que les parents de Lucile et de Camille ont des habitudes de sorties à tendances socialisantes hors du cadre familial proche avec de hauts résultats concernant les vacances, le sport en club, les promenades, les restaurants, etc. Tout en ayant quelques habitudes littéraires.

Voyons à présent les habitudes des parents de Roxane :

Figure 2. Représentation des pratiques culturelles des parents de Roxane

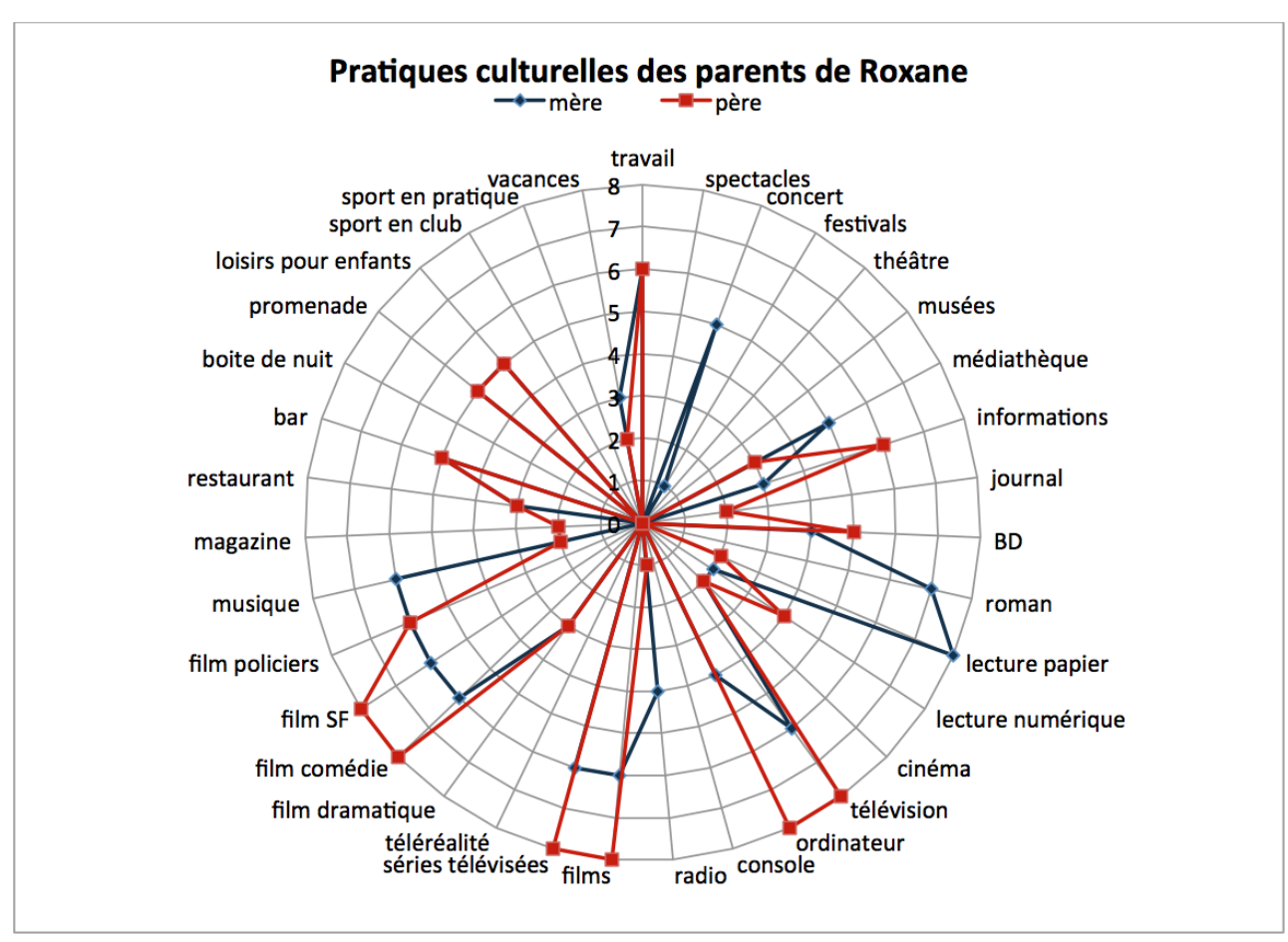



audiovisuelles, mais aussi la lecture. Les sorties à tendances sociales hors du cadre familial sont néanmoins présentes mais avec des résultats moins élevés que pour la famille précédente.

retiendrons que les pratiques culturelles de la famille de Camille et Lucile sont plus des activités en extérieur, à visée socialisante alors que les parents de Roxane sont sur des activités qui peuvent se pratiquer à l'intérieur même de leur foyer, qui ne sortent donc pas du cadre familial. Ces radars sont un extrait de ce que nous pouvons obtenir à l'aide de la fiche de renseignements, nous pourrons les croiser avec les résultats obtenus pour les usages des liaisons dans le corpus afin de voir dans quelle mesure l'usage des liaisons facultatives peut être influencé par les pratiques d'activités différentes. Ainsi, notre méthode permettra de croiser des représentations issues du questionnaire et des pratiques à partir de l'usage des liaisons.

\subsection{Panel de locuteurs}

La contrainte consistant à diversifier les familles sur le plan socioculturel a influencé notre façon d'aborder les prises de contact. Il est évident que les CSP défavorisées restent les familles les plus difficiles à atteindre. En effet, différentes caractéristiques de notre protocole peuvent freiner tout particulièrement les locuteurs appartenant à des CSP défavorisées : sur l'éducation qu'ils donnent à leur(s) enfant(s) (Neill, 1966 ; Gagnier, 2011),

l'enregistrement de mineurs et de leur milieu intime que constitue la famille (Linard \& Prax, 1984).

ous ces points compliquent la façon d'approcher ces locuteurs, et d'obtenir leur accord. Nous avons alors mis en place différents modes d'approche.

L'un d'eux a consisté à nous rapprocher d'une école élémentaire dont nous connaissions la directrice et où la proportion d'enfants issus de familles de CSP basses est importante. Nous sommes entrée directement en contact avec les familles lors d'une présentation orale du projet au moment de la réunion de rentrée dans les deux classes de $\mathrm{CP}$. Ce processus a permis d'avoir un plus grand impact sur les familles que de simplement leur donner un prospectus récapitulatif. Nous avons obtenu par ce biais l'accord de la famille de Marise.

L'autre moyen d'accéder à une diversité de familles a été d'utiliser nos réseaux, ce qui nous a permis d'enregistrer la famille de Fréîhr et Briséis (réseau proche) et la famille de Roxane (réseau plus distant). Les deux dernières participantes, Lucile et Camille, sont les filles d'une enseignante chercheuse participant au corpus ESLO. 


\begin{tabular}{|c|c|c|c|c|c|}
\hline $\begin{array}{l}\text { Enfant } \\
\text { étudié }\end{array}$ & $\begin{array}{l}\text { Age } \\
\text { période 1 }\end{array}$ & $\begin{array}{l}\text { Age } \\
\text { période 2 }\end{array}$ & $\begin{array}{l}\text { Age } \\
\text { période } 3\end{array}$ & \multicolumn{2}{|c|}{ Membres de la famille } \\
\hline Marise & $6 ; 8$ & $7 ; 5$ & Non disponible & $\begin{array}{l}\text { - Mère } \\
\text { - Père }\end{array}$ & - Sœur ainée \\
\hline Fréïhr & $1 ; 11$ & $2 ; 10$ & Non disponible & $\begin{array}{l}\text { - Mère } \\
\text { - Père }\end{array}$ & $\begin{array}{l}\text { - Sœur Briséis } \\
\text { - Sœur ainée }\end{array}$ \\
\hline Briséis & $7 ; 1$ & $7 ; 11$ & Non disponible & $\begin{array}{l}\text { - Mère } \\
\text { - Père }\end{array}$ & $\begin{array}{l}\text { - Frère cadet Fréïhr } \\
\text { - Sœur ainée }\end{array}$ \\
\hline Roxane & $3 ; 10$ & $4 ; 8$ & $5 ; 3$ & $\begin{array}{l}\text { - Mère } \\
\text { - Père }\end{array}$ & - Sœur cadette \\
\hline Lucile & $3 ; 9$ & $4 ; 9$ & $5 ; 2$ & $\begin{array}{l}\text { - Mère } \\
\text { - Père }\end{array}$ & - Sœur ainée (Camille) \\
\hline Camille & $6 ; 11$ & $7 ; 10$ & $8 ; 3$ & $\begin{array}{l}\text { - Mère } \\
\text { - Père }\end{array}$ & - Sœur cadette (Lucile) \\
\hline
\end{tabular}

\subsection{Formulaire de consentement / aspects juridiques / éthiques} procédons à deux types d'enregistrements. Le premier est un enregistrement vidéo qui permettra de mieux comprendre les scènes puisqu'avec un public enfantin, il est souvent nécessaire de visualiser la scène pour savoir à qui l'enfant parle et de quoi il parle. Pour cela, nous choisissons d'utiliser une caméra d'action car elle possède un champ d'enregistrement large et parce qu'elle est très petite, ce qui permet d'être moins intrusif quant à l'intimité des familles (Linard \& Prax, 1984). Nous espérons, ainsi, réduire le "paradoxe de l'observateur" (Labov, 1972). Le second est un enregistrement audio pour suivre au mieux les paroles des participants principaux. Nous choisissons d'utiliser deux micros-cravates HF sans fil qui seront portés par l'enfant et un membre de la famille.

Afin de réduire l'effet du "paradoxe de l'observateur » et d'accéder aux situations les plus écologiques possibles, nous ne sommes pas présente lors des enregistrements. Notre absence permettra aux familles de rester alors dans un cadre intime, de leur quotidien qui permet d'obtenir des données dans leur contexte social ordinaire et routinier (Mondada, 2012). Nous fournissons le kit d'enregistrement et le mode 
opératoire aux familles, avec des fiches de suivi que les familles devront remplir ${ }^{1}$ pour chaque enregistrement effectué. Dans ces fiches, nous demandons de noter les participants à l'enregistrement, la date, le lieu et ce qui se passe durant l'enregistrement. Les familles peuvent préciser s'il y a des passages qu'elles souhaiteraient ne pas voir apparaître dans le corpus. Ces informations serviront à remplir les métadonnées des enregistrements.

En ce qui concerne le rythme de collecte des enregistrements, nous tentons de réduire le temps d'emprunt du matériel par les familles, ce qui n'est pas toujours aisé. Nous laissons tout de même des libertés aux familles pour ne pas qu'elles se sentent pressées, ni oppressées par nos demandes. Cette démarche permet de pas ou peu impacter le comportement des familles pour la réalisation des enregistrements mais présente l'inconvénient de ne pas avoir des temps de collecte homogènes d'une famille à l'autre. Nous demandons aux familles d'enregistrer au moins 4 scènes par période représentant des moments clefs de la vie de leurs enfants, tels que les repas, les moments de jeux, les devoirs, le rituel de la douche, etc. avec les personnes que les enfants côtoient fréquemment.

Voici les caractéristiques des enregistrements récoltés pour la première et la deuxième période :

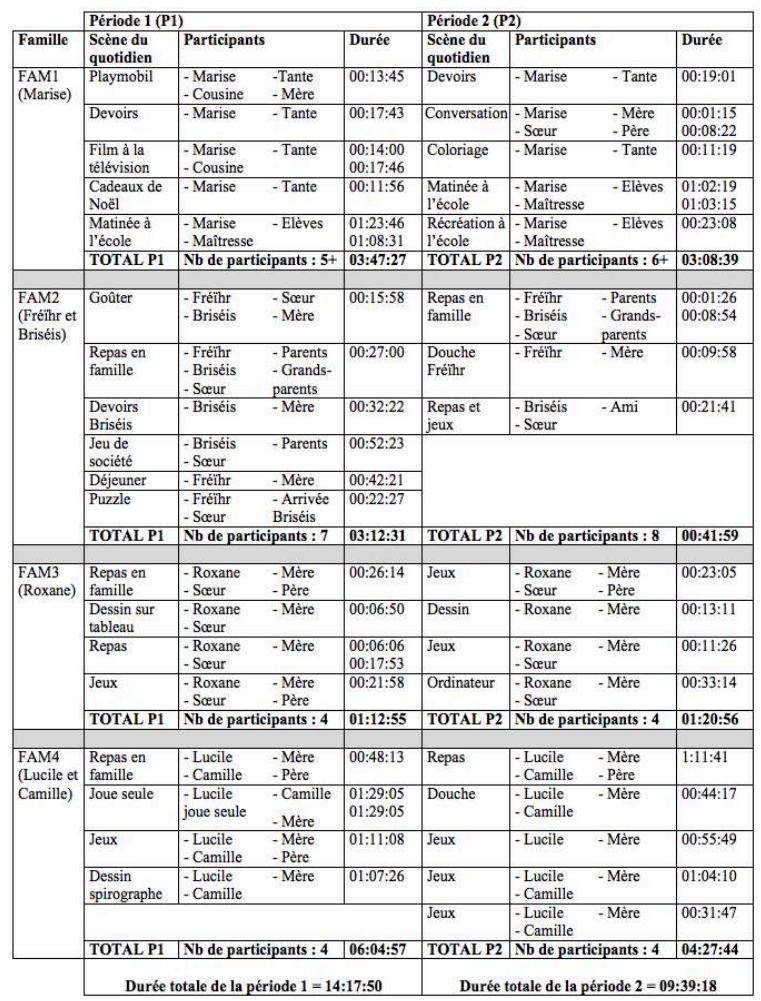

56 Nous obtenons une première période de $14 \mathrm{~h} 17$ et une deuxième période de $9 \mathrm{~h} 39$. La différence de durée peut s'expliquer par la non-maitrise du matériel de la part des familles. En effet, deux familles ont eu des difficultés à se servir de l'enregistreur et/ou la caméra lors de la deuxième période. Nous obtenons alors des enregistrements plus courts que prévu.

Les types de situations recueillis sont relativement variés: des repas (goûter, repas de famille), différents types de jeux (solitaires, jeux de sociétés, dessins, puzzle), des temps scolaires, les devoirs, des bains/douches. Par ailleurs, d'une période à l'autre, nous 
recueillons des scènes relativement semblables ce qui va nous permettre une comparaison intéressante.

Les enregistrements du quotidien ne sont pas les seules données langagières que nous récoltons. Des tests de langage sont effectués pour situer les capacités langagières de notre échantillon mais aussi pour provoquer des contextes de liaisons similaires d'une période à l'autre.

\subsection{Tests de langage}

59 Nous effectuons deux séries de tests, la première composée de tests de langage pour situer l'enfant dans son acquisition langagière et une deuxième série pour évaluer la manière dont la liaison se met en place. Pour cette dernière série, nous présenterons des résultats préliminaires de trois enfants : Roxane, Lucile et Camille (les trois enfants pour lesquels nous avons toutes les données sur les trois périodes).

\subsubsection{Tests de positionnement}

À la suite de chaque période d'enregistrements, les enfants ont effectué des tests de langage issus de DyLNet (Nardy et al. 2016). Il s'agit de vérifier l'étendue du lexique de l'enfant, de contrôler sa mémoire des chiffres, de connaître ses compétences sociolinguistiques en perception et en production, de voir s'il parvient à détecter les variations stylistiques en fonction des différents interlocuteurs possibles et enfin de connaître la compréhension syntaxicosémantique. Nous réalisons ces tests avec les enfants de notre panel les plus petits (Fréïhr, Briséis, Roxane, Lucile et Marise) puisque ces tests ont été construits pour le niveau " maternelle». Nos données pourront être comparées aux données DyLNet, recueillies sur toute une école maternelle de Grenoble.

\subsubsection{Tests de liaison}

\subsubsection{Pour tous les enfants}

61 La deuxième série de tests (empruntée à Dugua, 2006) porte sur la production de liaisons. En premier lieu, nous effectuons une tâche d'apostrophe où les enfants doivent appeler, à deux reprises et les uns après les autres, des animaux en disant par exemple « ours ours viens jouer avec moi ! ».

62 Cette tâche a pour but de voir la forme lexicale la plus disponible pour les enfants : à voyelle initiale «ours» ([urs]) ou à consonne initiale «nours» ([nuss]) issu de [œ̃nuss], «tours » ([tubs]) issu de [pətituss]. Les résultats laissent apparaître que les trois enfants aux périodes 2 et 3 , produisent les formes à voyelle initiale. Pour la période 1 (voir Figure 3), Lucile dispose clairement de la forme à voyelle initiale. Pour Camille, la production avec déterminant correspond à la forme [pətitan] avec liaison, mais nous supposons qu'elle possède la forme à initiale vocalique puisqu'elle a produit [an] en isolation lors de la seconde production. Quant à Roxane, nous voyons que la forme à initiale vocalique n'est pas forcément acquise, les formes avec déterminant sont en l'+ « nom » ([lekyвœj] et [lan]). 
Figure 3. Production de la tâche d'apostrophe (Période 1)

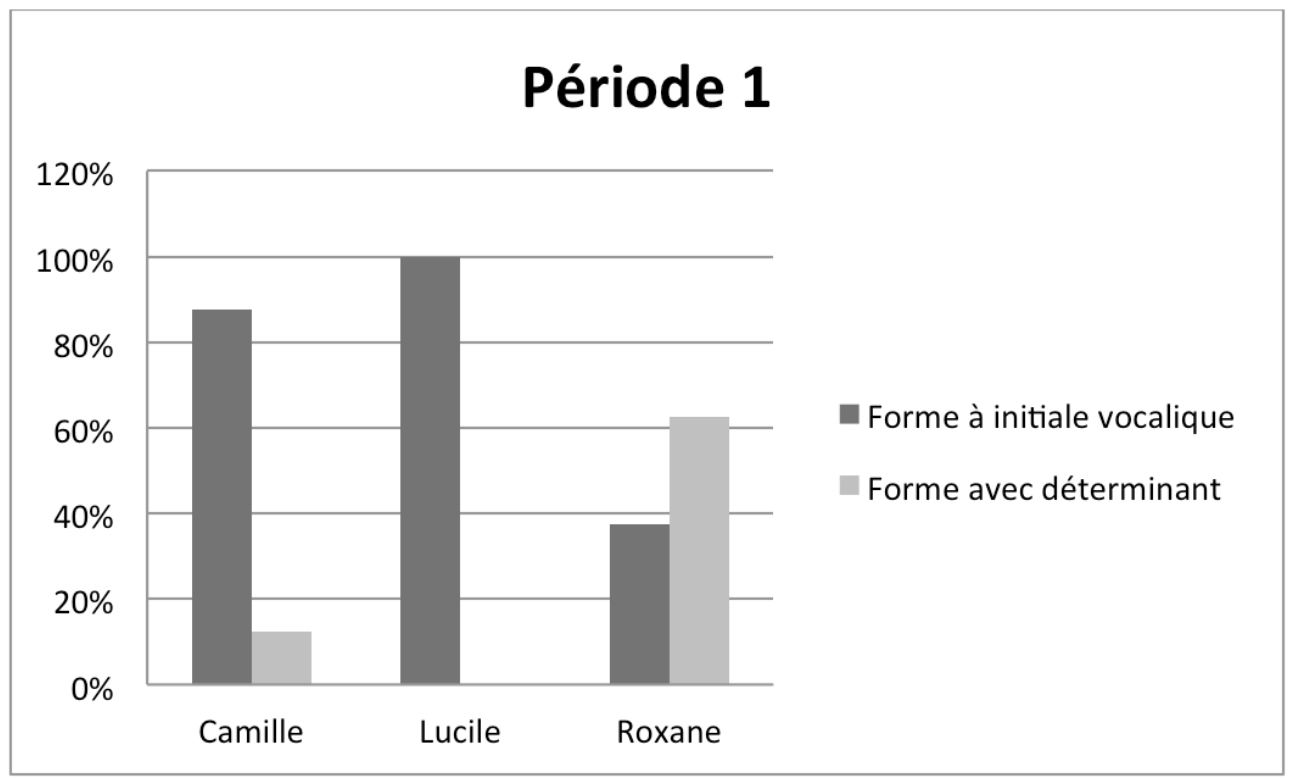

Dans un second temps, nous faisons une tâche avec des pseudo-noms à voyelle initiale (4 pseudo-noms), où nous présentons chaque pseudo-nom précédé du déterminant « un », par exemple [õnikat], l'enfant doit alors produire la forme « deux » + « pseudonom », par exemple [døzikat]. Nous relevons trois types de productions :

- Production de type maintien : nous disons [õnivak] l'enfant produit [dønivak]

- Production de type alternance : nous disons [œ̃nivak] l'enfant produit [døzivak]

- Production de type omission : nous disons [œ̃nivak] l'enfant produit [døivak]

67 Le graphique ci-dessous rend compte de la répartition des types de productions (4 productions au total) pour chaque enfant et à chaque période.

Figure 4. Résultats de la tâche de pseudo-noms

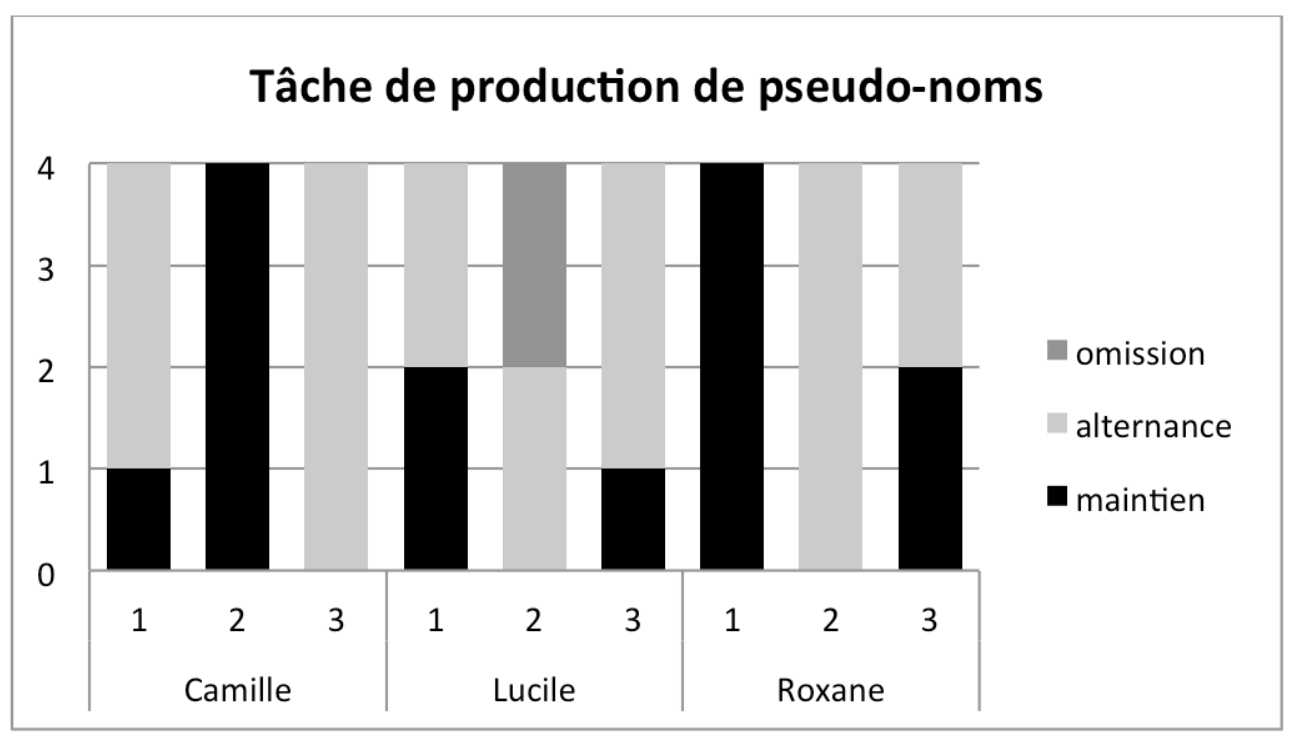




\section{Figure 5. Résultats de la tâche de dénomination de liaisons obligatoires}

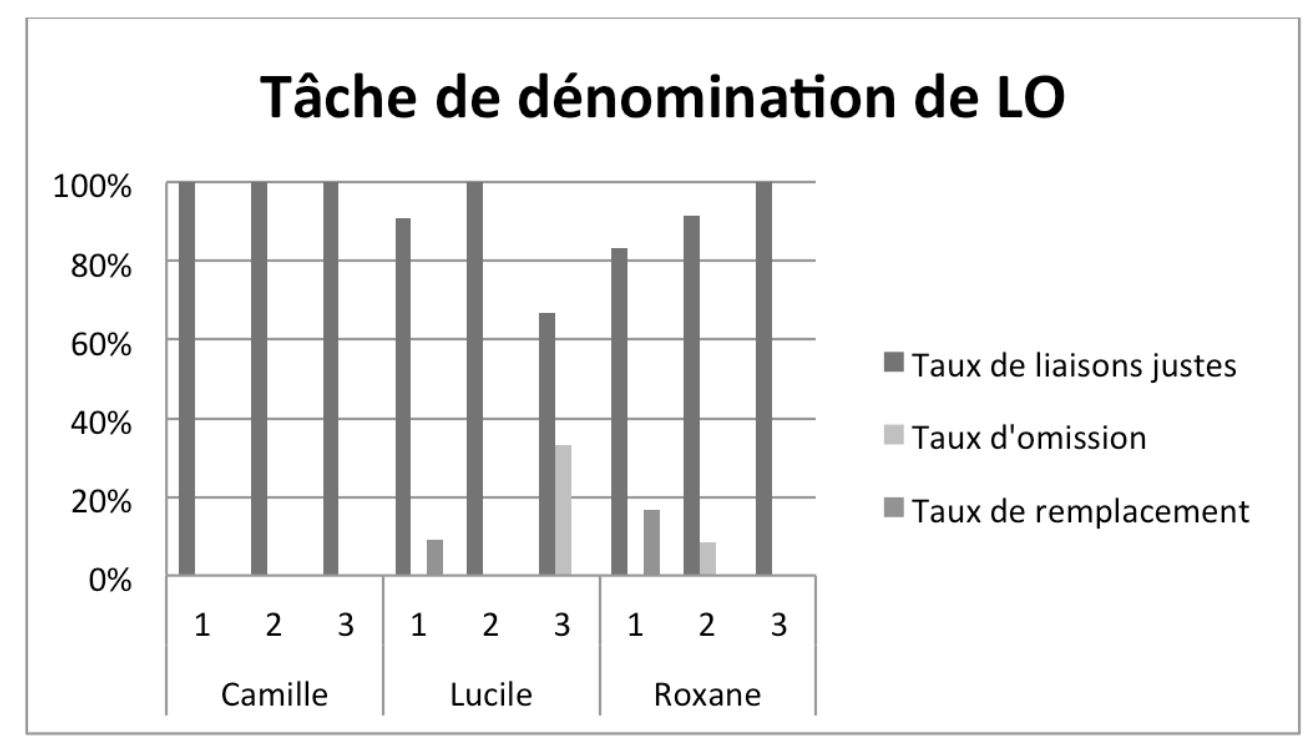

Les trois enfants observées présentent des comportements variables sur les formes d'alternances et de maintien que nous ne pouvons interpréter pour l'instant. Seule Lucile produit des formes en omissions lors de la deuxième période.

Par la suite, nous effectuons une tâche de dénomination d'images en contexte de liaisons obligatoires après les Mots 1 «un» et «deux», par exemple [õnurs]. Trois types de production sont attendus :

- Après « un » : [õnubs]

- Après « deux » : [døzurs]

- Production de type remplacement :

- Après « un » : [õzurs]

- Après « deux » : [dønurs]

- Production de type omission :

- Après « un » : [õurs]

- Après « deux » : [døuss]

Le graphique ci-dessous montre les taux de chaque type de production pour chaque enfant et à chacune des trois périodes.

\section{Tâche de dénomination de LO}

Pour Camille, qui est plus âgée, nous retrouvons des productions de liaisons justes systématiquement pour les trois périodes. Tandis que pour les plus petites, Lucile et Roxane, nous relevons des erreurs par remplacement lors de la première période, qui disparaissent par la suite. Des erreurs d'omission surviennent en période 2 et/ou 3, ce sont des erreurs qui peuvent subsister jusqu'à 6 ans (Dugua, 2006). Ces résultats sont en accord avec les données dont nous disposons sur les productions de liaisons.

Dans le même principe, la tâche suivante est également une dénomination d'images mais cette fois en contexte de liaison facultative après les Mots1 "petit » et «gros ». Trois types de productions sont attendus :

76 - Production de type liaison réalisée :

- Après « petit » : [pətiturs] 
- Après « gros » : [gвоzuвs]

- Production de type non réalisée (après les LF les liaisons ne sont pas obligatoires, la non production de liaison n'est donc pas une erreur) :

- Après « petit » : [pətiuss]

- Après « gros » : [gвоuвs]

\section{pour chaque enfant et à chacune des trois périodes.}

Figure 6. Résultats de la tâche de dénomination de liaisons facultatives

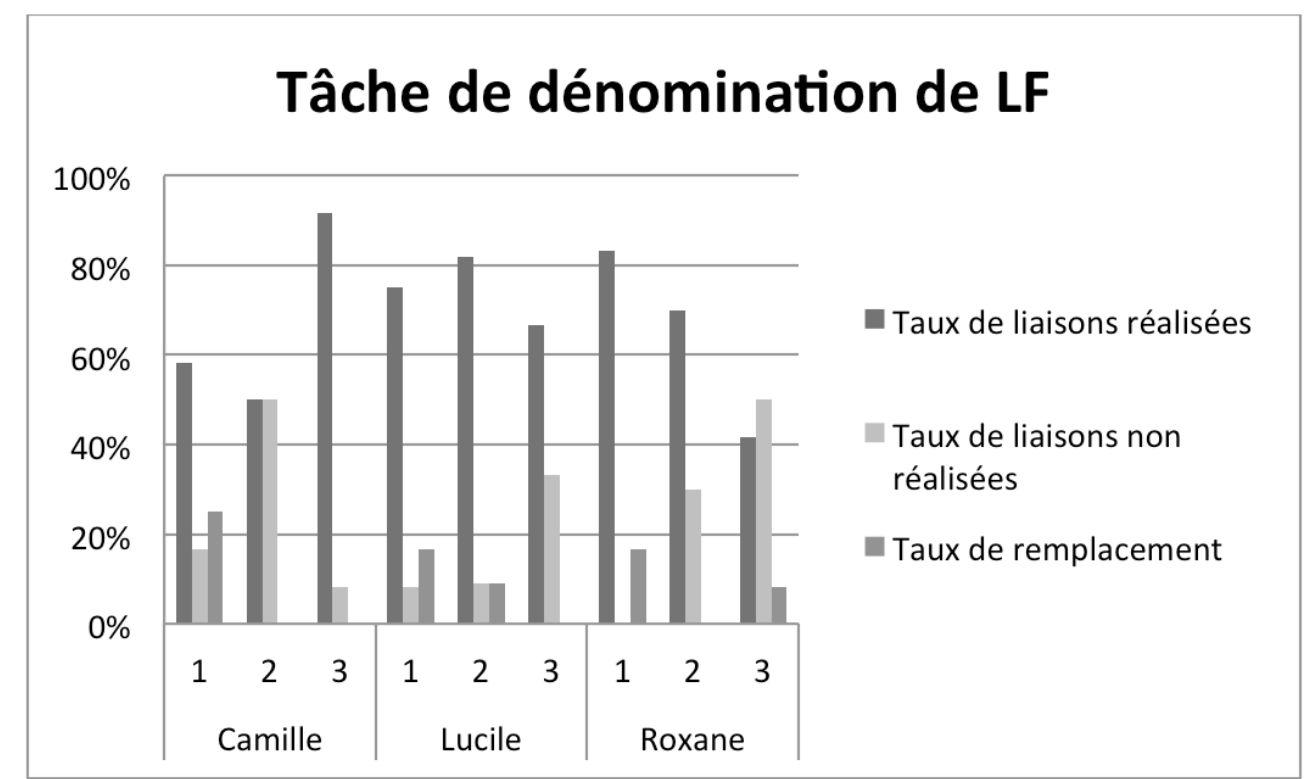

D'après ces résultats, nous retrouvons globalement des taux de réalisations de LF nettement supérieurs à Dugua (2006), nous sommes ici en moyenne à $69 \%$ de liaisons réalisées contre 20,5\% relevées chez Dugua (2006). Nous constatons des comportements bien différents chez les trois enfants : des taux de réalisation relativement stables et hauts pour Lucile, ces mêmes taux qui baissent pour Roxane et qui stagnent puis augmentent fortement pour Camille. L'analyse de ces résultats sera approfondie par la suite.

Enfin, la dernière tâche consiste à faire dénommer quatre noms à initiale consonantique (" nombril », " lavabo ", « nuage ", "zèbre ») après « un » et " deux ». Ces mots présentent la particularité d'avoir en initiale une consonne qui pourrait être une consonne liaison ou d'élision. Chez nos trois enfants, quand elles produisent les mots cibles, elles les réalisent correctement. Seules deux occurrences en période 1 présentent des variations intéressantes: [œ̃navabo] (Roxane) et [døzõbril] (Lucile). Dans ces erreurs, la consonne initiale produite correspond à la consonne de liaison attendue après les déterminants « un » et " deux ». Dugua (2006) interprète ce type de réalisation comme des surgénéralisations du phénomène de liaison. Dans ses données, elles apparaissent principalement vers 4-5 ans. Nous les retrouvons un an plus tôt chez Lucile et Roxane 

diversité et la richesse des inputs dans lesquels les enfants évoluent. À travers l'étude de l'usage des liaisons à la fois obligatoires et variables, dans la parole environnante, comme dans la parole enfantine, nous chercherons à mettre en évidence les liens entre l'usage des parents/fratrie/famille et l'usage des enfants, et la façon dont adultes et enfants adaptent leurs usages à la situation et aux participants. Des entretiens semidirectifs ont été réalisés (mais n'ont pas été présentés ici par manque de place) et permettront d'avoir un regard plus approfondi sur la vie au sein des familles, mais également d'avoir accès à l'usage langagier des parents dans un contexte plus formel que les scènes quotidiennes. La méthode que nous mettrons en place prendra deux orientations : (1) étant donné l'échantillon important recueilli, transcrit et annoté en liaisons, nous pourrons dégager des tendances statistiques (taux de liaisons selon les situations, selon les participants, etc.). Mais, (2) dans le but de rendre compte des influences de l'input sur l'output, il nous semble indispensable d'observer les usages des liaisons avec une approche plus qualitative en repérant, par exemple, des constructions fréquentes dans une famille et en cherchant à voir comment elles sont transmises et reprises ou non par les enfants. Ces deux méthodes d'observation et d'analyse, croisées avec les métadonnées sur les familles d'une part (avec l'échelle 
SLCP), et sur le développement langagier des enfants d'autre part (avec les tests de langage) devraient nous permettre de mieux comprendre la façon dont le langage se construit dans la diversité des environnements et des situations que rencontre un enfant.

\section{BIBLIOGRAPHIE}

Abouda L. \& Baude O. (2005). « Constituer et exploiter un grand corpus oral : choix et enjeux théoriques. Le cas des ESLO ", Corpus en Lettres et Sciences sociales, Des documents numériques à l'interprétation. Albi, France.

Baude O. \& Dugua C. (2011). « (Re)faire le corpus d'Orléans quarante ans après : quoi de neuf, linguiste? » Corpus $10: 99-118$.

Baude O. \& Dugua C. (2015). « Usage de la liaison dans le corpus des ESLOs : vers de nouveaux (z) ouvrages de référence ? » Dostie, Hedermann (éd.), La dia-variation en français actuel. Bern : Peter Lang, 349-371.

Blanc M. \& Biggs P. (1971). «L'enquête socio-linguistique sur le français parlé à Orléans »Le français dans le monde $85: 16-25$.

Bourdieu P. (1982). Ce que parler veut dire : L'économie des échanges linguistiques. Fayard.

Bourdieu P. (1994). Raisons pratiques : sur la théorie de l'action. Paris : éd. du Seuil.

Bourdieu P. (1979). La distinction. Critique sociale du jugement. Paris : éd. de Minuit.

Bybee J. \& Hopper P. (2001). «Introduction ». Bybee J. \& Hopper P. (éd.), Frequency and the Emergence of Linguistic Structure. Amsterdam/Philadelphia : Benjamins.

Cameron-Faulkner T., Lieven E. \& Tomasello M. (2003). « A construction based analysis of child directed speech ». Cognitive Science 27-6 : 843-873.

Chevrot J.-P., Chabanal D. \& Dugua C. (2007). « Pour un modèle de l'acquisition des liaisons basé sur l'usage : trois études de cas » Journal of French Language Studies 17, 103-128.

Chevrot J.-P., Dugua C. \& Fayol M. (2009). « Liaison Acquisition, Word Segmentation and Construction » Fench : A Usage Based Account. Journal of Child Language 36, 557-596.

Coquillon A., Durand J. \& Eychenne J. (2010). « La liaison dans PFC : les chiffres et leur interprétation » Atelier PFC. University Tulane, New Orleans., Jul 2010, Nouvelle Orléans, ÉtatsUnis.

De Jong D. (1994). « La sociophonologie de la liaison orléanaise ». Lyche C. (éd.), French Generative Phonology : Retrospective and Perspectives. Salford : ESRI, 95-129.

Dugua C. (2006). «Liaison, segmentation lexicale et schémas syntaxiques entre 2 et 6 ans - Un modèle développemental basé sur l'usage ». Thèse de doctorat, Université Stendhal, Grenoble.

Dugua C. \& Baude O. (2017). « La liaison à Orléans, corpus et changement linguistique : une première étude exploratoire ", Journal of French Language Studies 27, 41-54. 
Durand J. \& Lyche C. (2008). «French liaison in the light of corpus data » French Language Studies 18, 33-66.

Durand J., Laks B., Calderone B. \& Tchobanov A. (2011). « Que savons-nous de la liaison aujourd'hui ». Langue française 169, 103-135.

Encrevé P. (1988). La liaison avec et sans enchainement : phonologie tridimensionnelle et usages du français. Paris : Seuil.

Fijalkow J. (1997). « Entrer dans l'écrit : oui, mais par quelle porte? ». Repères, recherches en didactique du français langue maternelle 15, 113-129.

Gadet F. (1989). Le Français ordinaire. Paris : Armand Colin.

Gadet F. (1997). « Classe sociale ». M.L. Moreau (dir.). Sociolinguistique. Concepts de base. Liège : Mardaga.

Gagnier N. (2011). « Le jugement des autres sur nos attitudes parentales ». La Presse. Mis en ligne le 22 mars 2011. Consulté le 27 juillet 2019.

Goldin-Meadow S. (2010). « Rôle des gestes dans la création et l'acquisition du langage. » Enfance $62-3: 347-360$.

Institut National de la Statistique et des Etudes Economiques (2019). https://www.insee.fr/fr/ metadonnees/pcs2003/categorieSocioprofessionnelleAgregee/1

Kemmer S. \& Barlow M. (2000). «Introduction : A usage-based conception of language ». Barlow M. \& Kemmer S. (éd.), Usage-based models of language use. Stanford Californie : CSLI Publications, 7-28.

Labov W. (1976). Sociolinguistique. Paris : Editions de Minuit.

Laks B. (2005). « La liaison et l'illusion » Langages 39e année, 158 : 101-125.

Liégeois L. (2014). «Usage des variables phonologiques dans un corpus d'interactions naturelles parents-enfant : impact du bain linguistique et dispositifs cognitifs d'apprentissage » Thèse de doctorat, Université Blaise Pascal, Clermont 2.

Linard M. \& Prax I. (1984). Images vidéo, images de soi ou Narcisse au travail. Paris : Dunod.

Mondada L. (2012). « The conversation analytic approach to data collection ». J. Sidnell \& T. Stivers (éd.) Handbook of Conversation Analysis. Oxford : Blackwell-Wiley.

Nardy A., Fleury É., Chevrot J.-P., Karsai M., Buson L., Bianco M., Rousset I., Dugua C., Liégeois L., Barbu S., Crespelle C., Busson A., Léo Y. \& Bouchet H. (2016). DyLNet - Language Dynamics, Linguistic Learning, and Sociability at Preschool : Benefits of Wireless Proximity Sensors in Collecting Big Data (ANR-16-CE28-0013). https://dylnet.univ-grenoble-alpes.fr/

Neill A.S. (1966). La Liberté, pas l'anarchie. Hart Publishing (New York) 1966, Payot.

Queneau R. (1950). L’Avenir du français, Bâtons, chiffres et lettres. Paris : Gallimard.

Soum-Favaro C., Coquillon A. \& Chevrot J.-P. (2014). La liaison : approches contemporaines. Bern : Peter Lang.

Tomasello M. (2003). Constructing a Language : A Usage-Based Theory of Language Acqusition. Harvard University Press.

Référence des corpus : 
Corpus de Lyon : http://childes.psy.cmu.edu/access/French/Lyon.html

Laboratoire Ligérien de Linguistique - UMR 7270 (LLL) (2017). ESLO [Corpus]. ORTOLANG (Open Resources and TOols for LANGuage) - www.ortolang.fr, https://hdl.handle.net/11403/eslo/v1.

Laboratoire de Recherche sur le Langage - EA 999 (LRL) (2017). ALIPE (Acquisition de la Liaison et Interactions Parents Enfants) [Corpus]. ORTOLANG (Open Resources and TOols for LANGuage) www.ortolang.fr, https://hdl.handle.net/11403/alipe-000853/v1.1.

Communication Langagière chez le Jeune Enfant (CoLaJE) : http://colaje.scicog.fr/

Morgenstern, A \& Parisse, C. (2012). The Paris Corpus. French Language Studies 22(1), 7-12, Cambridge University Press.

Parisse C., Morgenstern A. (2010). Transcrire et analyser les corpus d'interactions adulte-enfant. Edy Veneziano, Anne Salazar Orvig, Josie Bernicot. Acquisition du langage et interaction, L'Harmattan, 201-222.

Traitement des Corpus Oraux en Français (TCOF) : http://www.cnrtl.fr/corpus/tcof/ Emmanuelle Canut, Virginie André, Bertrand Gaiffe. Mise à disposition de corpus oraux interactifs : le projet TCOF (Traitement des Corpus Oraux en Français). Pratiques : théorie, pratique, pédagogie, 2010, Interactions et Corpus Oraux, pp.147-148. <hal-00523397>

\section{NOTES}

1. Ces informations auraient pu être enregistrées si nous avions rencontré des familles dans l'impossibilité de remplir la fiche.

\section{RÉSUMÉS}

Le module ESLO-Enfants est un corpus variationniste longitudinal issu du grand corpus de langue française : Enquêtes SocioLinguistiques à Orléans (ESLO). Variationniste car il s'appuie sur un public varié (enfants de 2 ans à 7 ans avec leur entourage proche) provenant de familles de différentes catégories socio-économico-culturelles et enregistré dans des situations naturelles variées formant le quotidien des enfants. Ce corpus, qui sera mis à disposition à la fois sur ESLO et Childes, a été créé dans le but d'étudier l'impact de l'environnement langagier (input) sur les productions enfantines (output). Pour cette étude, la liaison, objet à la fois stable et variable, s'est révélée être un phénomène sociolinguistique propice pour observer sa transmission à des enfants en cours d'acquisition du langage mais aussi pour observer d'éventuelles différences entre les familles.

The ESLO-Children module is a longitudinal variationist corpus derived from the large Frenchlanguage corpus: Enquêtes SocioLinguistiques à Orléans (ESLO). It is variationist because it is based on a varied audience (children aged 2 to 7 with their close relatives) from families of different socio-economic and cultural categories and recorded in a variety of natural situations that form the daily lives of children. This corpus, which will be made available on both ESLO and Childes, was created with the aim of studying the impact of the language environment (input) on 
children's productions (ouput). For this study, liaison, an object that is both stable and variable, has been shown to be a sociolinguistic phenomenon that is conducive to observing its transmission on children in the process of language acquisition, but also to observing possible differences between families.

INDEX

Mots-clés : Acquisition du langage, corpus variationniste, liaison, ESLO, parole enfantine, situation naturelle

Keywords : Language acquisition, variationist corpus, liaison, ESLO, children's speech, natural situation

AUTEUR

JENNIFER GANAYE

LLL - UMR7270, Université Orléans 